\title{
Ny Varo-Mandeha Amin'Ny Faritra Manodidina Ny Alan'Analalava
}

\author{
Fidèle Raharimalala
}

\section{Fampidirana}

Analalava dia ala kely lavalava iray hita amin'ny sarintan'i Madagasikara, eo ho eo amin'ny $8 \mathrm{~km}$ andrefan'i tanànan'i Mahavelona (Foulpointe), faritany Toamasina. Ny tanjon'ity fanadihadiana ity dia fitadiavana hevitra hampivoarana miaraka ny fiainan'ny olona sy ny tontolo manodidina azy ho fitandrovana ny ala sy ny tontolo iainana. . Tsara ho marihina aloha fa ho an'ny malagasy dia ny vary ihany no heverina ho tena foto-tsakafo, na monina aiza izy na aiza, ka izay hahazoany izany vary hohanina izany, na amin'ny fomba inona, na manao ahoana no mahamay azy. Izany no anisan'ny mahapotika ny ala raha amin'iny faritra atsinana iny izay kely velaran-tany hambolena vary anaty rano. Ity fanadihadiana ity dia mikasika ny fifandraisan'ny olona sy ny zavamaniry amin'ireo mponina manodidina azy.

\section{Fomba nentina nanao ny fanadihadiana}

Ny varo-mandeha no endrim-pifandraisana notrandrahana. Ny fomba nanatanterahana izany dia fandehanana tongotra nandritry ny adiny roa amin'ny lalam-be falehan'ny olona rehetra, manelanelana ny tanan'i Morarano sy Sataloto. Misy koa ireo fanontaniana napetraka tamin'ireo olona izay nifanena teny an-dalana; tsy nisy moa ny fanavahana fa dia nanontaniana daholo, izay olona rehetra nifanena taminay. Ireto ary ny karazam-panontaniana napetraka tamin'izany :

- Firy taona izy?

- Avy aiza ny fiaviany?

- Inona ny karazan'entana entiny?

- Firy ny habetsany na ny isany?

- Hamidy aiza?

- Taiza no nakana ny entana izay hamidiny?

- Mpamboly sa mpiantoka?

- Velarantany misy ny voly?

- Fanodinana natao raha nisy izany?

- Isaky ny fotoana inona no mandeha mivarotra?
- Inona avy no ampiasana ny vola azo amin'ny varotra?

- Efa firy taona no nanaovany toy izao?

\section{Vokatra}

Ny valin'ny fanadihadiana dia ahazoana mampisongadina ny asa amam-panaon'ny karazan'olona amin'ny toerana iray mandavan-taona sy mikajy ny fifandraisany amin'ny zavamaniry sy ny vokatr'izany eo amin'ny olona sy ny tontolo iainany. Aseho amin'ny tabilao 1 izany.

Tsangan'olona efatra amibin'ny folo (14) no nahazoana valiny ka azo tsinjaraina toy izao: 4 lehilahy lehibe mihoatra ny 40 taona; 3 vehivavy lehibe latsaky ny 40 taona; 4 tovolahy 18 - 25 taona; 2 tovovavy 18 - 25 taona; ary ankizy kely efatra mianadahy niaraka nokaontina olona 1. Ny tabilao 2 manaraka dia mampiseho ny fifandraisan'ny zavamaniry sy ny olona araka ny fampiasana azy.

\section{Correspondence}

Fidèle Raharimalala, Département Botanique, Université d'Antananarivo, BP 906, Antananarivo 101, MADAGASIKARA. cofirah@antananarivo.mg

Ethnobotany Research \& Applications 3:335-337(2005) 
Tabilao 1. Famintinanany asa natao manodidina ny alan'Analalava. (LL: Lehilahy, VV: Vehivavy)

\begin{tabular}{|l|l|l|l|}
\hline Karazana Asa & Mpanao Asa & Fisokajiana ny karazan'asa & Fotoana fanaovana azy \\
\hline Voly vary & LL + VV & $\begin{array}{l}\text { An-Koraka, } \\
\text { An-tavy }\end{array}$ & $\begin{array}{l}\text { Aogositra - desambra } \\
\text { Mamafy latsak'orana }\end{array}$ \\
\hline Rary & VV & $\begin{array}{l}\text { Tanty bazary, Tanty be, } \\
\text { Tapatsihy, Tandroho }\end{array}$ & Rehefa tsy misy asa vary \\
\hline Varo -mandeha & LL & $\begin{array}{l}\text { Sakafo (voasary, sakoa, akondro, soanambo ) } \\
\text { Akanjo (tee-shirt, zipo, short) }\end{array}$ & Rehefa vita asa vary \\
\hline Mitanika toaka & LL & & $\begin{array}{l}\text { Mandava-taona ivelan'ny } \\
\text { asa vary }\end{array}$ \\
\hline Miompy kisoa & LL + VV & & Mandava-taona \\
\hline Voly hafa & VV & $\begin{array}{l}\text { Voly mangahazo, } \\
\text { Voly tsomanga, } \\
\text { Voly anana (Petsay, ,Anamalaho, baranjely, karoty) }\end{array}$ & $\begin{array}{l}\text { Manomboka septambra, } \\
\text { Manomboka Avril, }\end{array}$ \\
\hline Tsenakely & LL +VV & $\begin{array}{l}\text { Jiro, sira, afo, savony, } \\
\text { kafe ranony, betsa }\end{array}$ & mandavataona \\
\hline
\end{tabular}

\section{Kirakiran-kevitra}

Azo tsoahina avy amin'ireo tabilao roa ireo fa :

- Ny vary no tena fototr'asan'ny olona, fa ny sisa rehetra fanampiny fotsiny.

- Ny vola azo avy amin'ireo asa hafa dia entina hividianana vary satria ny vokatra azo tsy mahampy taona, ary hividianana kojakojam-piainana andavanandro. Ohatra ny vola azo amin'ny fivarotana tanty bazary dia $1000 \mathrm{Ar}$ isan-kerinandro ho an'ny vehivavy iray (tanty 10) ary $800 \mathrm{Ar}$ ho an'ny tovovavy iray (tanty 8). Lehilahy iray dia mahazo $20.000 \mathrm{Ar}$ amin'ny fivarotana toaka 20 litatra. Ny ankamaroan'ny mpivaro-mandeha dia avy any Andondambe $(20 \mathrm{~km}$ avy Mahavelona) sy Ambodifaho. Ny ankamaroan'ny mpividy dia eo Morarano.

Hita koa fa tsy misy miala avy anaty ala ny zavatra ampiasain'ny olona amin'ny fiainany andavanandro. Voamarina izany ny filazany fa tsy ry zareo manakaiky ny ala loatra no manapotika ny ala fa olona avy any ivelany: mponin'ny Mahavelona sy Toamasina. Mazava ihany koa, fa raha mahita vaha-olana mahafa-po an-dry zareo dia tsy hitetika ny ala koa ry zareo hambolem-bary an-tanety.
Efa nisy sosokevitra nampitain'ny mponina manamorona ny ala fa raha misy barrage na toho-drano dia mbola manana tany malalaka azo atao tanimbary hampitomboany ny voka-bary ry zareo. Izay no valin'ny fanadihadina vita noho ny tsy fahampian'ny fotoana.

\section{Fisaorana}

Koa dia hisaorana ny Mpikarakara rehetra, Missouri Botanical Garden sy Karoka Nasionaly Ampiharana ny Rakim-Panafody nahafahana nanatanteraka iny atrik'asa mikasika ny fifandraisan'ny 'olona sy ny zavamaniry. Misaotra ireo Mpampiantra avy any amin'ny Université of Hawaï nahafoy tena sy andro nanome ny fahalalana, isaorana koa ireo mpandray anjara avy eto an-toerana nanisy ambiansy tsara nandritry izany fotoana rehetra niarahana..

Tabilao 2. Fifandraisan'ny zavamanirysy ny olona araka ny famipasana azy.

\begin{tabular}{|l|l|l|l|}
\hline $\begin{array}{l}\text { Asa fanampiny } \\
\text { atao }\end{array}$ & $\begin{array}{l}\text { Zavamaniry fototra } \\
\text { (matières premières) }\end{array}$ & Toerana hakana azy & Habetsahan'ny alaina \\
\hline Rary & Penjy - arefo - zozoro & Honahona & 1 entana /olona \\
\hline Varo-mandeha & Lamba, Voankazo & Toamasina an-tsaha Andondabe & $\begin{array}{l}\text { Araka ny vola } \\
\text { Izay zaka mandeha tongotra }\end{array}$ \\
\hline Mitanika toaka & $\begin{array}{l}\text { Fary } \\
\text { akondro }\end{array}$ & $\begin{array}{l}\text { an-tsaha Andondabe } \\
\text { an-tsaha Andondabe }\end{array}$ & $\begin{array}{l}1 \text { taonina } \\
10 \text { reny }\end{array}$ \\
\hline Miompy kisoa & Viha & Honahona, any10km miala Morarano & $50 \mathrm{~kg}$ zaka bisikileta \\
\hline
\end{tabular}




\section{F. Raharimalala - Ny Varo-Mandeha Amin'Ny Faritra Manodidina Ny}

Alan'Analalava
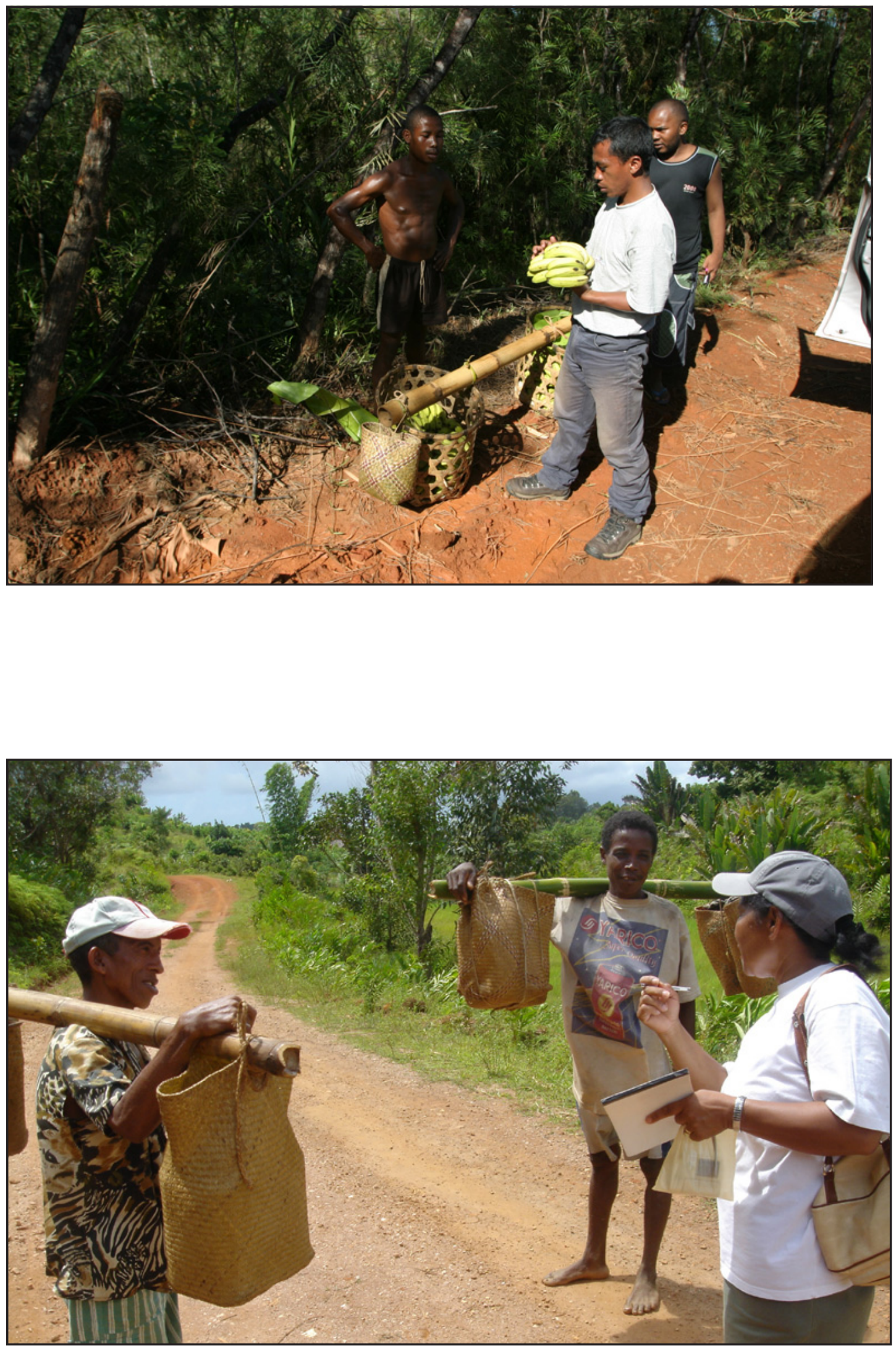

www.ethnobotanyjournal.org/vol3/i1547-3465-03-335.pdf 
